\title{
Therapeutic Outcomes in High-Grade B-Cell Lymphoma, NOS: Retrospective Analysis
}

\author{
Parathan Karunakaran ${ }^{1}$ Gangothri Selvarajan ${ }^{10} \quad$ Jayachandran Perumal Kalaiyarasi ${ }^{1}$ Nikita Mehra ${ }^{1}$ \\ Shirley Sundersingh ${ }^{2}$ Manikandan Dhanushkodi ${ }^{1}$ Sivasree Kesana ${ }^{1}$ Krishnarathinam Kannan ${ }^{1}$ \\ Trivadi S. Ganesan ${ }^{1}$ Venkatraman Radhakrishnan ${ }^{1}$ Tenali Gnana Sagar ${ }^{1}$
}

${ }^{1}$ Department of Medical Oncology, Cancer Institute (WIA), Chennai, India

2 Department of Onco-Pathology, Cancer Institute (WIA), Chennai, India

Address for correspondence Gangothri Selvarajan, MD, DM, Department of Medical Oncology, Cancer Institute (WIA), Adyar, Chennai 600036, India (e-mail: gangothri11@gmail.com).

\section{South Asian J Cancer 2022;11(1):68-72.}

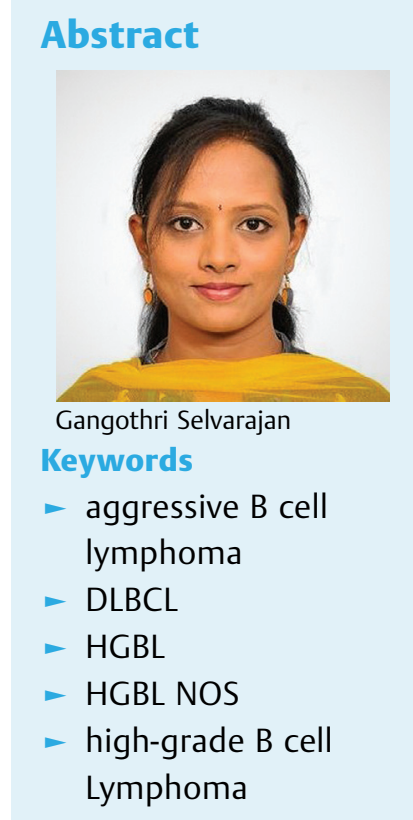

The nomenclature high-grade non-Hodgkin's lymphoma was repurposed in the World Health Organization (WHO) 2016 update as high-grade B cell lymphoma (HGBL). However, among the HGBL entities HGBL, not otherwise specified (NOS) remains a poorly described entity with a lack of literature regarding its treatment and prognosis. The baseline characteristics, treatment, and outcome of HGBL, NOS cases were analyzed. Thirty HGBL, NOS patients were diagnosed between January 2017 and December 2019. Their median age was 49.3 years, and $30 \%$ had advanced IPI. The majority received R-CHOP chemotherapy, while five patients received dose-adjusted R$\mathrm{EPOCH}$. At a median follow-up of 15 months, nine patients had disease progression or relapse. EFS and OS were 22 months (12.1-31.9 months) and 37 months (29.4-44.0 months) respectively. Only NCCN-IPI $\leq 2$ showed significant influence on the outcome. The results were similar to the outcomes previously reported.

This study highlights the importance of NCCN-IPI in ascertaining the prognosis of HGBL, NOS. The literature review suggests that more intensive chemotherapy is ideal for HGBL, NOS. However, prospective trials are needed to prove whether the treatment of HGBL, NOS can be tailored based on NCCN-IPI.

\section{Introduction}

High-grade B cell lymphoma (HGBL) is a new disease entity as per the World Health Organization (WHO) 2016 update. The name resulted from the re-purposing of an existing lymphoma entity-high-grade B cell non-Hodgkin's lymphoma. Highgrade lymphoma was traditionally used to describe morphologically aggressive lymphoma with many mitotic figures, a
How to cite this article: Karunakaran P, Selvarajan G, Kalaiyarasi JP, et al. Therapeutic Outcomes in High-Grade B-Cell Lymphoma, NOS: Retrospective Analysis South Asian J Cancer 2022;11(1): $68-72$. starry-sky pattern, and a high proliferation rate. The newly defined HGBL consisted of two entities-HGBL with a double hit (DH) or triple hit (TH) and HGBL, not otherwise specified (NOS). ${ }^{1}$ HGBL DH is defined based on the rearrangement of MYC and BCL2 or BCL6. HGBL TH is defined as the rearrangement of all the above three genes. The other entity in this category-HGBL, NOS was defined solely based on the morphology in the absence of the above genetic rearrangements. ${ }^{2}$

(c) 2022. MedIntel Services Pvt Ltd. All rights reserved.

This is an open access article published by Thieme under the terms of the Creative Commons Attribution-NonDerivative-NonCommercial-License, permitting copying and reproduction so long as the original work is given appropriate credit. Contents may not be used for commercial purposes, or adapted, remixed transformed or built upon. (https://creativecommons.org/licenses/by-nc-nd/ 4.0/)

Thieme Medical and Scientific Publishers Pvt. Ltd., A-12, 2nd Floor, Sector 2, Noida-201301 UP, India 
HGBL with $\mathrm{DH} / \mathrm{TH}$ has shown to have a poor prognosis compared with diffuse large B cell lymphoma (DLBCL). These $\mathrm{DH} / \mathrm{TH}$ lymphomas require more aggressive therapy than the standard R-CHOP regimen. In contrast, HGBL, NOS remains a poorly described entity. Four years after the WHO introduced the entity, there is a conspicuous lack of literature on this entity, with no definite guidelines for treating this entity. We performed this retrospective analysis to analyze the clinical characteristics and therapeutic outcomes of HGBL, NOS cases at our institute.

\section{Methods}

This study was a single-center, retrospective analysis of all patients diagnosed with HGBL, NOS from January 2017 to December 2019. We obtained ethical clearance from the Institutional Ethics Committee. Data were retrieved from the electronic records of patients diagnosed with HGBL, NOS. All patients aged over 18 years who received at least more than three planned chemotherapy cycles were included. High-grade transformations from indolent lymphomas were excluded.

The Eastern Cooperative Oncology Group performance status (ECOG-PS), baseline serum LDH, histopathology reports, FISH report for rearrangement of MYC, BCL2, and $B C L 6$, staging investigations, treatment details, response assessment, and outcomes were collected from the case records.

Diagnosis of HGBL, NOS was made based on the morphology and immunophenotypic features as described in the literature. ${ }^{3}$ Patients with gene rearrangement by FISH for $M Y C$ with BCL2 and/or BCL6 were excluded from the analysis. The choice of chemotherapy regimen was at the discretion of the individual physician. The chemotherapy regimen of RCHOP or dose-adjusted R-EPOCH was given as previously described in the literature. ${ }^{4,5}$ The response to therapy was evaluated upon completion of planned treatment based on the Lugano criteria. ${ }^{6}$

Non-achievement of complete response (CR) at the end of planned chemotherapy, relapse of the disease after achieving $\mathrm{CR}$, or death from any cause were defined as events. Eventfree survival (EFS) was calculated from the date of diagnosis to the occurrence of the event. Overall survival (OS) was calculated from the date of diagnosis to the death due to any cause or until the last follow-up. The data were censored in August 2020.

Baseline characteristics were reported as descriptive analysis. EFS and OS were plotted as Kaplan-Meier curves. Age, stage, LDH, International Prognostic Index (IPI), NCCNIPI, and type of chemotherapy were analyzed for prognostic significance by Cox regression. Univariate analysis was performed for each factor by log-rank test. Achieving a $p$-value of less than or equal to 0.05 was considered significant. The statistical analysis was performed with SPSS software version 21.

\section{Results}

Thirty patients were diagnosed with HGBL, NOS during the 3 -year study period. The median age was 49.3 years (range:
20-72 years). Sixteen patients (53.3\%) presented with advanced-stage disease (Stage III/IV). A high IPI score (3-5) was observed in nine patients (30\%) with a score $>1$ in $73.3 \%$. Among 30 patients, 20 (66.6\%) had an NCCN-IPI score of more than 2. The majority were with ECOG-PS 1 (22 patients, 73.3\%). Extra-nodal involvement was observed in 18 patients (60\%), while bone marrow and CNS involvement were observed in 4 patients and 1 patient, respectively. Twelve patients (40\%) presented with bulky disease, defined as any lymph node measuring $>7.5 \mathrm{~cm}$. The median $\mathrm{LDH}$ value was $632.0 \mathrm{U} / \mathrm{L}$ (range: 466.18-823.0), and the median $\mathrm{K}_{\mathrm{i}}-67$ value was $90 \%$ (range: 60-95\%). Among the 30 cases, 2 had isolated MYC positivity on FISH (negative for BCL2 and BCL6 rearrangement). Out of 30 patients, 25 received R-CHOP chemotherapy, while 5 patients received dose-adjusted REPOCH. The median number of chemotherapy cycles was 6 (range: $3-8$ cycles). Seven out of 30 patients (23.3\%) received consolidation radiotherapy to the initial site of bulky disease after attaining metabolic response. The baseline characteristics of the patients are presented in -Table 1.

The median follow-up of study subjects was 15 months (12.3-17.6 months). The median EFS was 22 months (12.131.9 months), and the median OS was 37 months (29.4-44.0 months). The Kaplan-Meier curves for overall EFS and OS are as shown in -Fig. 1. Among the 30 cases of HGBL, 9 had disease progression. One patient received DA R-EPOCH, while the other eight received R-CHOP. Among these 8 cases, four had primary progressive disease while undergoing chemotherapy. The remaining four had an early relapse, with a median event-free interval of only 6.5 months (range: 4-11 months). Out of nine patients, five received only metronomic therapy as they were not fit for further chemotherapy. Only four cases received salvage therapy with R-gemcitabine/oxaliplatin (R-GemOx) with or without radiotherapy. None of them could complete the planned six cycles of salvage therapy due to disease progression. None of the nine cases received an autologous stem cell transplant.

The univariate and multivariate analyses of variables affecting EFS and OS are as described in -Table 2. In univariate analysis, the NCCN-IPI score of $\leq 2$ demonstrated a statistically significant influence on EFS and OS $(p=0.013$ and $p=0.031$, respectively). Similarly, IPI score of $\leq 1$ influenced EFS $(p=0.03)$. However, with multivariate analysis, none of the factors influenced survival. - Fig. 2 shows the Kaplan-Meier curve analysis for OS and EFS for those with IPI score $\leq 1$ and $>1$, respectively, as well as for those with NCCN-IPI score $\leq 2$ and $>2$.

\section{Discussion}

The clinical profile and optimal therapy for HGBL, NOS have not been established. In general, clinical correlations from studies on HGBL, NOS are hampered by overlap with other lymphoma types and their retrospective nature. Numerous reports are available in the literature on B cell lymphoma, unclassifiable from which HGBL, NOS was derived. However, these reports were enriched with HGBL-DH and cannot be compared with HGBL, NOS. Since the WHO update in 2016, 
Table 1 Comparison of baseline characteristics of this study with published studies on HGBL, NOS

\begin{tabular}{|c|c|c|c|}
\hline & $\begin{array}{l}\text { Present } \\
\text { study }\end{array}$ & Li et $\mathrm{al}^{8}$ & Rush et $\mathrm{al}^{7}$ \\
\hline No. of cases & 30 & 41 & 50 \\
\hline Men & $73.3 \%$ & $63.4 \%$ & \\
\hline Age $>60 y$ & $26.6 \%$ & $26.8 \%$ & \\
\hline ECOG PS $>2$ & $03.3 \%$ & $31.7 \%$ & \\
\hline Stage III/IV & $53.3 \%$ & $56.1 \%$ & \\
\hline Extra nodal site $>1$ & $20.0 \%$ & $56.1 \%$ & \\
\hline $\mathrm{LDH}>\mathrm{ULN}$ & $76.66 \%$ & $61 \%$ & \\
\hline $\mathrm{LDH}>2 \mathrm{ULN}$ & $33.3 \%$ & & \\
\hline IPI score $>2$ & $30.0 \%$ & $58.5 \%$ & \\
\hline $\mid \mathrm{PI}>1$ & $73.3 \%$ & & \\
\hline NCCN-IPI $>2$ & $66.6 \%$ & & \\
\hline Ki67 > 90\% & $80.0 \%$ & $78 \%$ & \\
\hline $\begin{array}{l}\text { FISH-Myc } \\
\text { rearrangement } \\
\text { only }\end{array}$ & $06.6 \%$ & $39 \%$ & \\
\hline $\begin{array}{l}\text { Chemotherapy } \\
\text { R-CHOP } \\
\text { DA R-EPOCH }\end{array}$ & $\begin{array}{l}83.3 \% \\
16.6 \%\end{array}$ & $\begin{array}{l}41.5 \% \\
58.5 \%\end{array}$ & $\begin{array}{l}63 \% \\
37 \%{ }^{a}\end{array}$ \\
\hline $\begin{array}{l}\text { Outcome } \\
\text { - CR } \\
\text { - PR } \\
\text { - SD } \\
\text { - PD }\end{array}$ & $\begin{array}{l}76.6 \% \\
06.6 \% \\
03.3 \% \\
13.3 \%\end{array}$ & $\begin{array}{l}31.7 \% \\
26.8 \% \\
02.4 \% \\
39.0 \%\end{array}$ & \\
\hline Median OS & 37 months & 18 months & \\
\hline 2-year OS (\%) & $68 \%$ & & $76 \%$ \\
\hline
\end{tabular}

ancluded other intensive regimens.

Abbreviations: ULN, upper limit of normal; 2ULN, twice the ULN.

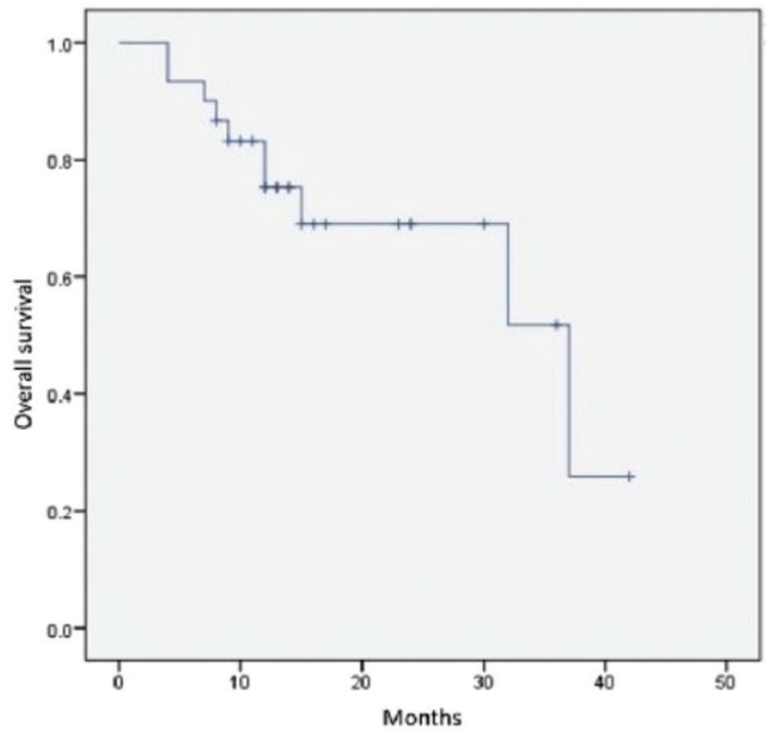

there have been only two retrospective studies on HGBL, NOS. Among the two, the report by Rush et al is available only as an abstract. ${ }^{7}$ Hence, we compared our data to 41 cases of HGBL, NOS from China by Li et al. ${ }^{8}$

The comparison of the clinical profiles along with baseline characters of these studies with the present study is shown in Table 1 . The overall outcome reported in our study was similar to that reported by Rush et al (2-year OS: 68\% vs. 76\%). However, in comparison to $\mathrm{Li}$ et al's study, a higher fraction of cases attained CR in this study ( $83.3 \%$ vs. $58.5 \%$ ). This disparity could be explained by the higher percentage of patients presenting with poor PS (31.7\% vs. 3.3\%) and higher IPI (58.5\% vs. 30\%) in their study.

The higher proportion of single-hit HGBL (only MYC positive) in Li et al's study (39\% vs. 6.6\%) may also have contributed to the lower CR rate. Interestingly. HGBL, NOS with MYC rearrangement alone (without BCL2 or BCL6) was classified as Burkitt's lymphoma in the WHO 2016 update, reaffirming the fact that they had a poor prognosis. ${ }^{2}$ However, this is not universally accepted, with some authors suggesting that these cases can still be classified as HGBL,

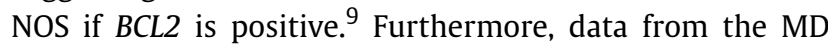
Anderson Cancer Centre suggests that this subset of HGBL benefits the most from intensive chemotherapy. ${ }^{10}$ Similarly, Li et al also demonstrated that isolated MYC positivity was associated with more inferior PFS and OS.

To summarize, HGBL covers three disease entities with varied prognoses. HGBL with isolated MYC positivity has the worst prognosis, followed by HGBL with DH/TH. ${ }^{11-14} \mathrm{HGBL}$, NOS (excluding MYC isolated positivity) has a relatively better prognosis although they still perform poorly in comparison to DLBCL, NOS. $3,9,11,12$

The therapy of HGBL with $\mathrm{DH} / \mathrm{TH}$ is well defined now with multiple studies showing benefits for more intensive

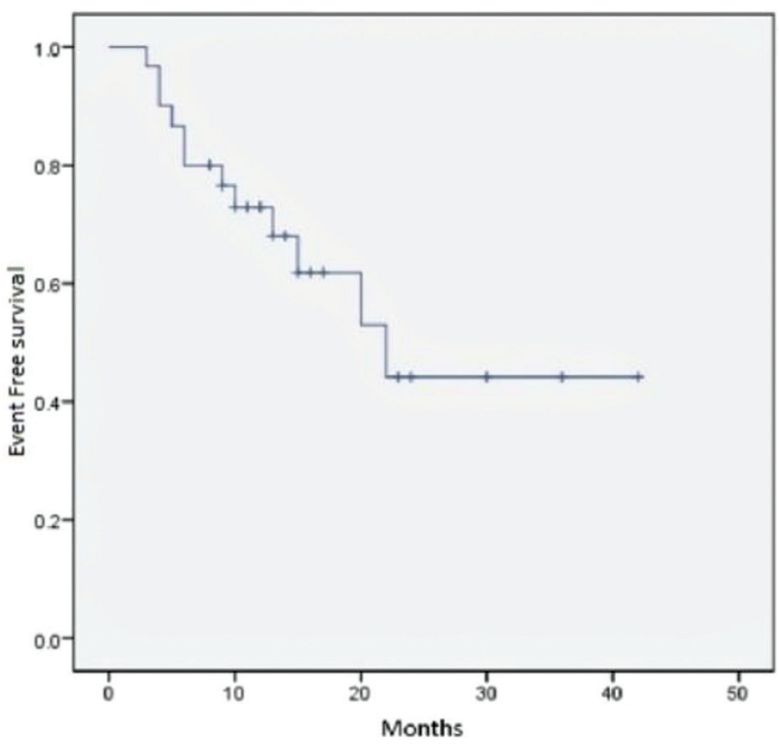

Fig. 1 Overall survival and event-free survival curves of all 30 patients. 
Table 2 Univariate and multivariate analyses of various factors for EFS and OS

\begin{tabular}{|l|l|l|l|l|}
\hline Variable & \multicolumn{2}{|l|}{$\begin{array}{l}\text { Univariate analysis (by log- } \\
\text { rank) } p \text {-value }\end{array}$} & \multicolumn{2}{l|}{$\begin{array}{l}\text { Multivariate analysis (by Cox- } \\
\text { regression) } p \text {-value }\end{array}$} \\
\hline & EFS & OS & \multicolumn{2}{l|}{ EFS } \\
\hline Age $(y)$ & 0.09 & 0.12 & 0.919 & 0.609 \\
\hline Stage $\leq 2$ vs. $>2$ & 0.445 & 0.083 & 0.119 & 0.81 \\
\hline LDH $\leq 2$ ULN vs. $>2$ ULN & 0.06 & 0.1 & 0.903 & 0.929 \\
\hline IPI $\leq 1$ vs. $>1$ & 0.03 & 0.057 & 0.994 & 0.990 \\
\hline NCCN-IPI $\leq 2$ vs. $>2$ & 0.013 & 0.031 & 0.973 & 0.980 \\
\hline Type of chemotherapy & 0.128 & 0.736 & 0.241 & 0.776 \\
\hline
\end{tabular}

Abbreviations: EFS, event-free survival; OS, overall survival.

protocols. ${ }^{7}$ Similarly, the NCCN guidelines have endorsed the need for more intensive protocols for HGBL NOS. ${ }^{15}$ In Li et al's study, nearly $60 \%$ of the patients received more intensive chemotherapy regimens. In our study, the patients who received dose-adjusted $\mathrm{R}$-EPOCH fared better than those who received R-CHOP although the difference in outcomes was not statistically significant. However, our data support using IPI or NCCN-IPI to determine the intensity of therapy, as
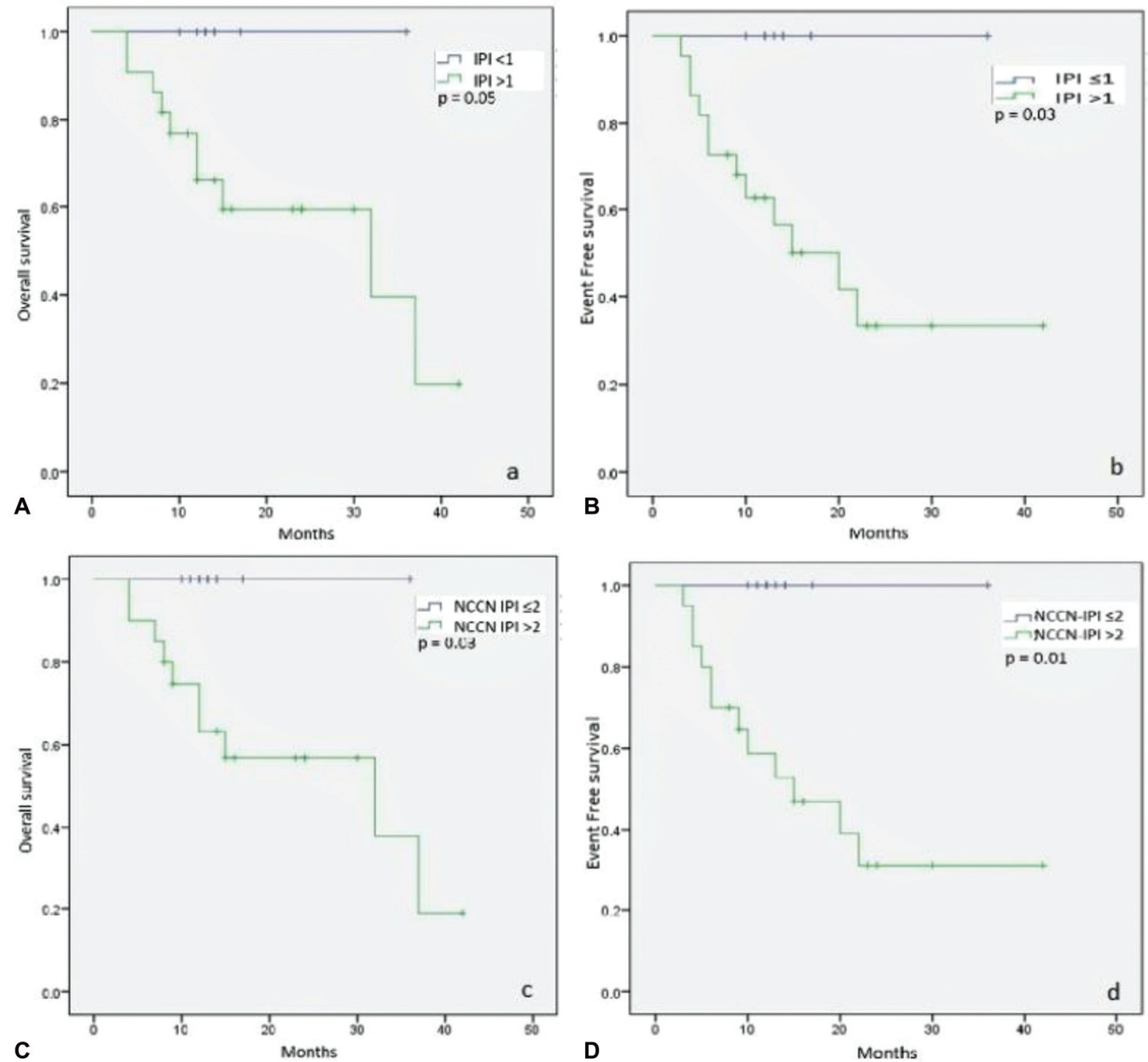

Fig. 2 (A and B) show overall survival and event-free survival curves for those with IPI score $\leq 1$ and $>1$, respectively. (C and D) show OS and EFS survival curves for those with NCCN-IPI score $\leq 2$ and $>2$, respectively. 
those with IPI $\leq 1$ or NCCN-IPI $\leq 2$ had an excellent outcome with R-CHOP alone.

None of the patients who relapsed in our study attained a second CR. The lack of response to salvage therapy with RGemOx seen in this study is similar to the poor real-world outcome reported in the literature. ${ }^{16}$ The lack of access to newer immunotherapy and chemotherapeutic modalities is reflected in the poor outcome among the cases with disease progression. This serves to underline that ideal upfront chemotherapy offers the best chance to cure a lymphoma patient in low- and middle-income countries.

In addition to the retrospective nature of this study, the other potential limitations of our study include the small sample size and the lack of a consistent chemotherapy regimen with dose-adjusted $\mathrm{R}-\mathrm{EPOCH}$ given in only five patients. Nevertheless, collectively, the data are hypothesis-generating and suggest a possible role for more intensive therapy such as R-EPOCH in HGBL, NOS in high IPI/NCCN-IPI or those with isolated MYC positivity.

\section{Conclusion}

HGBL, NOS is a heterogeneous subgroup of high-grade B cell Lymphoma. There is an unmet need to characterize HGBL, NOS without a high-risk MYC single hit. The intensive chemotherapy approach appears to be the ideal choice of therapy upfront. Prospective trials are needed to prove this hypothesis.

\section{Funding \\ None.}

\section{Conflict of Interest}

None declared.

\section{Acknowledgments}

We would like to thank Dr. Swaminathan (Department of Epidemiology and Tumour Registry) and his staff for assisting in data retrieval.

\section{Bibliography}

1 Chen BJ, Fend F, Campo E, Quintanilla-Martinez L. Aggressive B-cell lymphomas-from morphology to molecular pathogenesis. Ann Lymphoma 2019;3:1-22

2 Swerdlow SH, Campo E, Pileri SA, et al. The 2016 revision of the World Health Organization classification of lymphoid neoplasms. Blood 2016;127(20):2375-2390

3 Swerdlow SH, Campo E, Harris NL, et al. WHO Classification of Tumours of Haematopoietic and Lymphoid Tissues, revised. 4th ed. Vol. 2. Lyon: IARC; 2017

4 Coiffier B, Lepage E, Briere J, et al. CHOP chemotherapy plus rituximab compared with $\mathrm{CHOP}$ alone in elderly patients with diffuse large-B-cell lymphoma. N Engl J Med 2002;346(04): 235-242

5 Wilson WH, Dunleavy K, Pittaluga S, et al. Phase II study of dose-adjusted $\mathrm{EPOCH}$ and rituximab in untreated diffuse large B-cell lymphoma with analysis of germinal center and postgerminal center biomarkers. J Clin Oncol 2008;26(16): 2717-2724

6 Cheson BD, Fisher RI, Barrington SF, et al; Alliance, Australasian Leukaemia and Lymphoma Group Eastern Cooperative Oncology Group European Mantle Cell Lymphoma Consortium Italian Lymphoma Foundation European Organisation for Research Treatment of Cancer/Dutch Hemato-Oncology Group Grupo Español de Médula Ósea German High-Grade Lymphoma Study Group German Hodgkin's Study Group Japanese Lymphorra Study Group Lymphoma Study Association NCIC Clinical Trials Group Nordic Lymphoma Study Group Southwest Oncology Group United Kingdom National Cancer Research Institute. Recommendations for initial evaluation, staging, and response assessment of Hodgkin and non-Hodgkin lymphoma: the Lugano classification. J Clin Oncol 2014;32(27):3059-3068

7 Rush J, Sehgal AR, Roth CG, Boyiadzis M. The effect of therapy on high grade B cell lymphoma, not otherwise specified and outcomes in comparison with double hit lymphoma. Blood 2016;128 (22):4224-4224

8 Li J, Liu X, Yao Z, Zhang M. High-grade B-cell lymphomas, not otherwise specified: a study of 41 cases. Cancer Manag Res 2020; 12:1903-1912

9 Ok CY, Medeiros LJ. High-grade B-cell lymphoma: a term repurposed in the revised WHO classification. Pathology 2020;52 (01):68-77

10 Lin P, Dickason TJ, Fayad LE, et al. Prognostic value of MYC rearrangement in cases of B-cell lymphoma, unclassifiable, with features intermediate between diffuse large B-cell lymphoma and Burkitt lymphoma. Cancer 2012;118(06): 1566-1573

11 Cook JR, Goldman B, Tubbs RR, et al. Clinical significance of MYC expression and/or "high-grade" morphology in non-Burkitt, diffuse aggressive B-cell lymphomas: a SWOG S9704 correlative study. Am J Surg Pathol 2014;38(04):494-501

12 Perry AM, Crockett D, Dave BJ, et al. B-cell lymphoma, unclassifiable, with features intermediate between diffuse large B-cell lymphoma and burkitt lymphoma: study of 39 cases. Br J Haematol 2013;162(01):40-49

13 Pedersen MO, Gang AO, Clasen-Linde E, et al. Stratification by MYC expression has prognostic impact in MYC translocated B-cell lymphoma-Identifies a subgroup of patients with poor outcome. Eur J Haematol 2019;102(05):395-406

14 Landsburg DJ, Falkiewicz MK, Petrich AM, et al. Sole rearrangement but not amplification of MYC is associated with a poor prognosis in patients with diffuse large B cell lymphoma and B cell lymphoma unclassifiable. Br J Haematol 2016;175(04): 631-640

15 National Comprehensive Cancer Network. NCCN Clinical Practice Guidelines in Oncology: B-Cell Lymphomas. Version 1.2020. Published on January 22, 2020

16 Schade JR, Kim C, Drill E, et al. Retrospective analysis of gemcitabine and oxaliplatin (GemOx)-based treatment in patients with relapsed/refractory aggressive B-cell non-Hodgkin lymphoma. Blood 2019;134(Supplement_1)2904 